Phosphate Tether-Mediated Ring-Closing Metathesis for the Generation of P-Stereogenic, Z-

Configured Bicyclo[7.3.1]- and Bicyclo[8.3.1]phosphates

Jana L Markley, Soma Maitra, and Paul R. Hanson*

Department of Chemistry, University of Kansas, 1251 Wescoe Hall Drive, Lawrence, KS 66045-7582

Email: Paul R. Hanson - phanson@ku.edu.

phanson@ku.edu

Thermal Ellipsoid Plot for Compound 15

$S X-2$

Tabular X-ray Parameters/Settings and Bond Lengths and Angles for $\mathbf{1 5}$

$S X-3$ to $S X-5$

Thermal Ellipsoid Plot for Compound trans-33

$S X-6$

Tabular X-ray Parameters/Settings and Bond Lengths and Angles for trans-33

$S X-7$ to $S X-10$ 
Figure 1. Thermal ellipsoid plot for compound 15; thermal ellipsoids are set at a 50\% probability level.
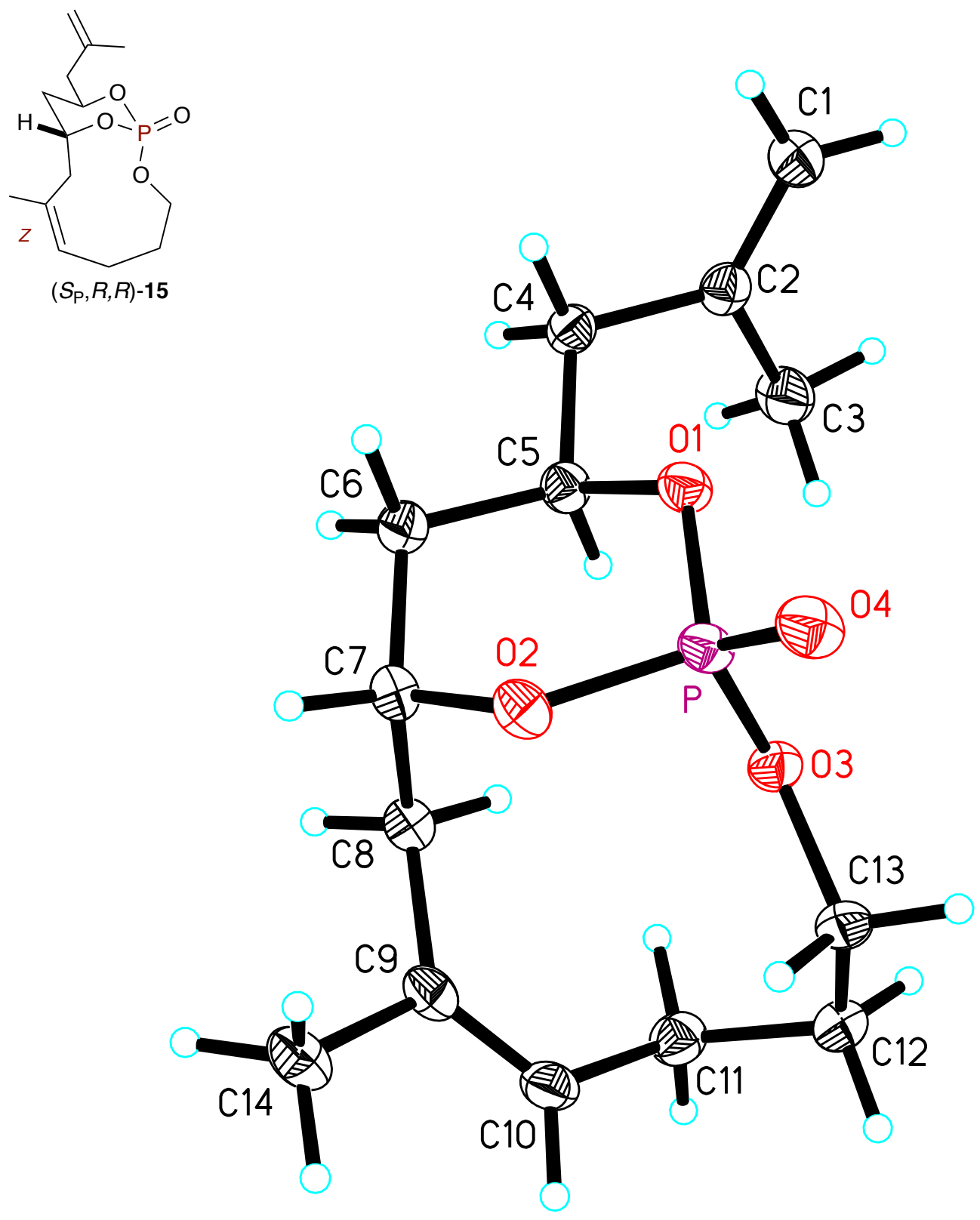
Table 1. Crystal data and structure refinement for Compound $\mathbf{1 5}\left(\mathrm{C}_{14} \mathrm{H}_{23} \mathrm{PO}_{4}\right)$

Identification code

Empirical formula

Formula weight

Temperature

Wavelength

Crystal system

Space group

Unit cell dimensions

Volume

$\mathrm{Z}$

Density (calculated)

Absorption coefficient

$\mathrm{F}(000)$

Crystal size

Theta range for data collection

Index ranges

Reflections collected

Independent reflections

Absorption correction

Max. and min. transmission

Refinement method

Data / restraints / parameters

Goodness-of-fit on $\mathrm{F}^{2}$

Final R indices [I $>2 \operatorname{sigma}(\mathrm{I})]$

$\mathrm{R}$ indices (all data)

Absolute structure parameter

Extinction coefficient

Largest diff. peak and hole

\section{Compound 15}

$\mathrm{C}_{14} \mathrm{H}_{23} \mathrm{PO}_{4}$

286.29

$100(2) \mathrm{K}$

$1.54178 \AA$

Monoclinic

P 21

$\mathrm{a}=7.9652(9) \AA \quad \alpha=90^{\circ}$

$\mathrm{b}=9.6983(10) \AA \quad \beta=99.7908(16)^{\circ}$

$\mathrm{c}=9.7159(10) \AA \quad \gamma=90^{\circ}$

739.61(14) $\AA^{3}$

2

$1.286 \mathrm{Mg} / \mathrm{m}^{3}$

$1.722 \mathrm{~mm}^{-1}$

308

$0.290 \times 0.240 \times 0.110 \mathrm{~mm}^{3}$

6.496 to $68.230^{\circ}$

$-9<=\mathrm{h}<=6,-11<=\mathrm{k}<=11,-11<=\mathrm{l}<=11$

8247

2415 [R(int) $=0.0354]$

Multi-scan

1.000 and 0.809

Full-matrix least-squares on $\mathrm{F}^{2}$

2415 / 1 / 265

1.093

$\mathrm{R} 1=0.0220, \mathrm{wR} 2=0.0562$

$\mathrm{R} 1=0.0220, \mathrm{wR} 2=0.0562$

$0.102(7)$

$0.0158(18)$

0.149 and -0.281 e. $\AA^{-3}$ 


\begin{tabular}{|c|c|c|c|}
\hline $\mathrm{P}-\mathrm{O}(4)$ & $1.4603(16)$ & $\mathrm{C}(12)-\mathrm{H}(12 \mathrm{~B})$ & $0.99(3)$ \\
\hline $\mathrm{P}-\mathrm{O}(1)$ & $1.5697(13)$ & $\mathrm{C}(13)-\mathrm{H}(13 \mathrm{~A})$ & $1.02(2)$ \\
\hline $\mathrm{P}-\mathrm{O}(3)$ & $1.5759(15)$ & $\mathrm{C}(13)-\mathrm{H}(13 \mathrm{~B})$ & $0.98(3)$ \\
\hline $\mathrm{P}-\mathrm{O}(2)$ & $1.5807(13)$ & $\mathrm{C}(14)-\mathrm{H}(14 \mathrm{~A})$ & $0.98(3)$ \\
\hline $\mathrm{O}(1)-\mathrm{C}(5)$ & $1.473(2)$ & $\mathrm{C}(14)-\mathrm{H}(14 \mathrm{~B})$ & $1.03(3)$ \\
\hline $\mathrm{O}(2)-\mathrm{C}(7)$ & $1.470(2)$ & $\mathrm{C}(14)-\mathrm{H}(14 \mathrm{C})$ & $0.95(3)$ \\
\hline $\mathrm{O}(3)-\mathrm{C}(13)$ & $1.468(2)$ & $\mathrm{O}(4)-\mathrm{P}-\mathrm{O}(1)$ & $113.83(9)$ \\
\hline$C(1)-C(2)$ & $1.324(3)$ & $\mathrm{O}(4)-\mathrm{P}-\mathrm{O}(3)$ & $116.04(7)$ \\
\hline $\mathrm{C}(1)-\mathrm{H}(1 \mathrm{~A})$ & $0.99(3)$ & $\mathrm{O}(1)-\mathrm{P}-\mathrm{O}(3)$ & $102.03(8)$ \\
\hline $\mathrm{C}(1)-\mathrm{H}(1 \mathrm{~B})$ & $0.97(3)$ & $\mathrm{O}(4)-\mathrm{P}-\mathrm{O}(2)$ & $111.11(9)$ \\
\hline$C(2)-C(3)$ & $1.496(3)$ & $\mathrm{O}(1)-\mathrm{P}-\mathrm{O}(2)$ & $105.69(7)$ \\
\hline$C(2)-C(4)$ & $1.513(3)$ & $\mathrm{O}(3)-\mathrm{P}-\mathrm{O}(2)$ & $107.24(8)$ \\
\hline $\mathrm{C}(3)-\mathrm{H}(3 \mathrm{~A})$ & $0.99(3)$ & $\mathrm{C}(5)-\mathrm{O}(1)-\mathrm{P}$ & $118.79(11)$ \\
\hline $\mathrm{C}(3)-\mathrm{H}(3 \mathrm{~B})$ & $0.92(3)$ & $\mathrm{C}(7)-\mathrm{O}(2)-\mathrm{P}$ & $121.50(12)$ \\
\hline $\mathrm{C}(3)-\mathrm{H}(3 \mathrm{C})$ & $1.03(3)$ & $\mathrm{C}(13)-\mathrm{O}(3)-\mathrm{P}$ & $118.99(13)$ \\
\hline$C(4)-C(5)$ & $1.520(3)$ & $\mathrm{C}(2)-\mathrm{C}(1)-\mathrm{H}(1 \mathrm{~A})$ & $120.5(18)$ \\
\hline $\mathrm{C}(4)-\mathrm{H}(4 \mathrm{~A})$ & $0.99(3)$ & $\mathrm{C}(2)-\mathrm{C}(1)-\mathrm{H}(1 \mathrm{~B})$ & $119.6(16)$ \\
\hline $\mathrm{C}(4)-\mathrm{H}(4 \mathrm{~B})$ & $0.98(3)$ & $\mathrm{H}(1 \mathrm{~A})-\mathrm{C}(1)-\mathrm{H}(1 \mathrm{~B})$ & $120(2)$ \\
\hline$C(5)-C(6)$ & $1.523(3)$ & $C(1)-C(2)-C(3)$ & $122.5(2)$ \\
\hline $\mathrm{C}(5)-\mathrm{H}(5)$ & $0.98(2)$ & $C(1)-C(2)-C(4)$ & $121.2(2)$ \\
\hline$C(6)-C(7)$ & $1.525(3)$ & $C(3)-C(2)-C(4)$ & $116.29(18)$ \\
\hline $\mathrm{C}(6)-\mathrm{H}(6 \mathrm{~A})$ & $0.98(3)$ & $\mathrm{C}(2)-\mathrm{C}(3)-\mathrm{H}(3 \mathrm{~A})$ & 108.1(18) \\
\hline $\mathrm{C}(6)-\mathrm{H}(6 \mathrm{~B})$ & $1.03(3)$ & $\mathrm{C}(2)-\mathrm{C}(3)-\mathrm{H}(3 \mathrm{~B})$ & $111.9(19)$ \\
\hline$C(7)-C(8)$ & $1.529(3)$ & $\mathrm{H}(3 \mathrm{~A})-\mathrm{C}(3)-\mathrm{H}(3 \mathrm{~B})$ & $110(3)$ \\
\hline $\mathrm{C}(7)-\mathrm{H}(7)$ & $0.98(3)$ & $\mathrm{C}(2)-\mathrm{C}(3)-\mathrm{H}(3 \mathrm{C})$ & $109.0(19)$ \\
\hline$C(8)-C(9)$ & $1.510(3)$ & $\mathrm{H}(3 \mathrm{~A})-\mathrm{C}(3)-\mathrm{H}(3 \mathrm{C})$ & $108(2)$ \\
\hline $\mathrm{C}(8)-\mathrm{H}(8 \mathrm{~A})$ & $0.98(2)$ & $\mathrm{H}(3 \mathrm{~B})-\mathrm{C}(3)-\mathrm{H}(3 \mathrm{C})$ & 109(3) \\
\hline $\mathrm{C}(8)-\mathrm{H}(8 \mathrm{~B})$ & $1.01(2)$ & $C(2)-C(4)-C(5)$ & $113.93(16)$ \\
\hline $\mathrm{C}(9)-\mathrm{C}(10)$ & $1.334(3)$ & $\mathrm{C}(2)-\mathrm{C}(4)-\mathrm{H}(4 \mathrm{~A})$ & 109.3(14) \\
\hline $\mathrm{C}(9)-\mathrm{C}(14)$ & $1.508(3)$ & $\mathrm{C}(5)-\mathrm{C}(4)-\mathrm{H}(4 \mathrm{~A})$ & $106.3(14)$ \\
\hline $\mathrm{C}(10)-\mathrm{C}(11)$ & $1.505(3)$ & $\mathrm{C}(2)-\mathrm{C}(4)-\mathrm{H}(4 \mathrm{~B})$ & $110.6(14)$ \\
\hline $\mathrm{C}(10)-\mathrm{H}(10)$ & $0.96(3)$ & $\mathrm{C}(5)-\mathrm{C}(4)-\mathrm{H}(4 \mathrm{~B})$ & 109.7(14) \\
\hline $\mathrm{C}(11)-\mathrm{C}(12)$ & $1.542(3)$ & $\mathrm{H}(4 \mathrm{~A})-\mathrm{C}(4)-\mathrm{H}(4 \mathrm{~B})$ & 106.6(19) \\
\hline $\mathrm{C}(11)-\mathrm{H}(11 \mathrm{~A})$ & $1.00(2)$ & $\mathrm{O}(1)-\mathrm{C}(5)-\mathrm{C}(4)$ & $107.02(16)$ \\
\hline $\mathrm{C}(11)-\mathrm{H}(11 \mathrm{~B})$ & $0.98(3)$ & $\mathrm{O}(1)-\mathrm{C}(5)-\mathrm{C}(6)$ & $108.58(16)$ \\
\hline $\mathrm{C}(12)-\mathrm{C}(13)$ & $1.508(3)$ & $C(4)-C(5)-C(6)$ & $112.97(16)$ \\
\hline $\mathrm{C}(12)-\mathrm{H}(12 \mathrm{~A})$ & $0.94(3)$ & $\mathrm{O}(1)-\mathrm{C}(5)-\mathrm{H}(5)$ & $107.1(12)$ \\
\hline
\end{tabular}




\begin{tabular}{|c|c|c|c|}
\hline $\mathrm{C}(4)-\mathrm{C}(5)-\mathrm{H}(5)$ & $109.8(12)$ & $\mathrm{O}(3)-\mathrm{C}(13)-\mathrm{H}(13 \mathrm{~A})$ & $109.2(13)$ \\
\hline $\mathrm{C}(6)-\mathrm{C}(5)-\mathrm{H}(5)$ & 111.1(12) & $\mathrm{C}(12)-\mathrm{C}(13)-\mathrm{H}(13 \mathrm{~A})$ & $112.9(13)$ \\
\hline$C(5)-C(6)-C(7)$ & $112.94(17)$ & $\mathrm{O}(3)-\mathrm{C}(13)-\mathrm{H}(13 \mathrm{~B})$ & $107.6(14)$ \\
\hline $\mathrm{C}(5)-\mathrm{C}(6)-\mathrm{H}(6 \mathrm{~A})$ & $108.2(14)$ & $\mathrm{C}(12)-\mathrm{C}(13)-\mathrm{H}(13 \mathrm{~B})$ & $109.5(14)$ \\
\hline $\mathrm{C}(7)-\mathrm{C}(6)-\mathrm{H}(6 \mathrm{~A})$ & $109.4(14)$ & $\mathrm{H}(13 \mathrm{~A})-\mathrm{C}(13)-\mathrm{H}(13 \mathrm{~B})$ & $108.8(19)$ \\
\hline $\mathrm{C}(5)-\mathrm{C}(6)-\mathrm{H}(6 \mathrm{~B})$ & $109.2(13)$ & $\mathrm{C}(9)-\mathrm{C}(14)-\mathrm{H}(14 \mathrm{~A})$ & $111.0(17)$ \\
\hline $\mathrm{C}(7)-\mathrm{C}(6)-\mathrm{H}(6 \mathrm{~B})$ & $108.9(14)$ & $\mathrm{C}(9)-\mathrm{C}(14)-\mathrm{H}(14 \mathrm{~B})$ & 111.3(16) \\
\hline $\mathrm{H}(6 \mathrm{~A})-\mathrm{C}(6)-\mathrm{H}(6 \mathrm{~B})$ & $108(2)$ & $\mathrm{H}(14 \mathrm{~A})-\mathrm{C}(14)-\mathrm{H}(14 \mathrm{~B})$ & $109(2)$ \\
\hline $\mathrm{O}(2)-\mathrm{C}(7)-\mathrm{C}(6)$ & $109.90(16)$ & $\mathrm{C}(9)-\mathrm{C}(14)-\mathrm{H}(14 \mathrm{C})$ & $113.3(16)$ \\
\hline $\mathrm{O}(2)-\mathrm{C}(7)-\mathrm{C}(8)$ & $111.62(16)$ & $\mathrm{H}(14 \mathrm{~A})-\mathrm{C}(14)-\mathrm{H}(14 \mathrm{C})$ & $104(2)$ \\
\hline$C(6)-C(7)-C(8)$ & $113.16(17)$ & $\mathrm{H}(14 \mathrm{~B})-\mathrm{C}(14)-\mathrm{H}(14 \mathrm{C})$ & $108(2)$ \\
\hline $\mathrm{O}(2)-\mathrm{C}(7)-\mathrm{H}(7)$ & $104.8(15)$ & & \\
\hline $\mathrm{C}(6)-\mathrm{C}(7)-\mathrm{H}(7)$ & $108.9(14)$ & & \\
\hline $\mathrm{C}(8)-\mathrm{C}(7)-\mathrm{H}(7)$ & $108.2(16)$ & & \\
\hline $\mathrm{C}(9)-\mathrm{C}(8)-\mathrm{C}(7)$ & $114.84(17)$ & & \\
\hline $\mathrm{C}(9)-\mathrm{C}(8)-\mathrm{H}(8 \mathrm{~A})$ & $108.5(13)$ & & \\
\hline $\mathrm{C}(7)-\mathrm{C}(8)-\mathrm{H}(8 \mathrm{~A})$ & $107.5(13)$ & & \\
\hline $\mathrm{C}(9)-\mathrm{C}(8)-\mathrm{H}(8 \mathrm{~B})$ & $109.4(13)$ & & \\
\hline $\mathrm{C}(7)-\mathrm{C}(8)-\mathrm{H}(8 \mathrm{~B})$ & $109.7(14)$ & & \\
\hline $\mathrm{H}(8 \mathrm{~A})-\mathrm{C}(8)-\mathrm{H}(8 \mathrm{~B})$ & $106.6(19)$ & & \\
\hline$C(10)-C(9)-C(14)$ & $120.75(19)$ & & \\
\hline$C(10)-C(9)-C(8)$ & $123.54(18)$ & & \\
\hline $\mathrm{C}(14)-\mathrm{C}(9)-\mathrm{C}(8)$ & $115.69(18)$ & & \\
\hline $\mathrm{C}(9)-\mathrm{C}(10)-\mathrm{C}(11)$ & $127.16(19)$ & & \\
\hline $\mathrm{C}(9)-\mathrm{C}(10)-\mathrm{H}(10)$ & $118.3(16)$ & & \\
\hline $\mathrm{C}(11)-\mathrm{C}(10)-\mathrm{H}(10)$ & $114.5(16)$ & & \\
\hline$C(10)-C(11)-C(12)$ & $112.52(19)$ & & \\
\hline $\mathrm{C}(10)-\mathrm{C}(11)-\mathrm{H}(11 \mathrm{~A})$ & $111.7(14)$ & & \\
\hline $\mathrm{C}(12)-\mathrm{C}(11)-\mathrm{H}(11 \mathrm{~A})$ & $106.7(14)$ & & \\
\hline $\mathrm{C}(10)-\mathrm{C}(11)-\mathrm{H}(11 \mathrm{~B})$ & $110.7(15)$ & & \\
\hline $\mathrm{C}(12)-\mathrm{C}(11)-\mathrm{H}(11 \mathrm{~B})$ & $109.7(15)$ & & \\
\hline $\mathrm{H}(11 \mathrm{~A})-\mathrm{C}(11)-\mathrm{H}(11 \mathrm{~B})$ & $105(2)$ & & \\
\hline$C(13)-C(12)-C(11)$ & $113.41(19)$ & & \\
\hline $\mathrm{C}(13)-\mathrm{C}(12)-\mathrm{H}(12 \mathrm{~A})$ & $104.9(18)$ & & \\
\hline$C(11)-C(12)-H(12 A)$ & $109.9(16)$ & & \\
\hline $\mathrm{C}(13)-\mathrm{C}(12)-\mathrm{H}(12 \mathrm{~B})$ & $110.7(16)$ & & \\
\hline $\mathrm{C}(11)-\mathrm{C}(12)-\mathrm{H}(12 \mathrm{~B})$ & $107.6(16)$ & & \\
\hline $\mathrm{H}(12 \mathrm{~A})-\mathrm{C}(12)-\mathrm{H}(12 \mathrm{~B})$ & $110(2)$ & & \\
\hline $\mathrm{O}(3)-\mathrm{C}(13)-\mathrm{C}(12)$ & $108.76(17)$ & & \\
\hline
\end{tabular}


Figure 2. Thermal ellipsoid plot for compound trans-33; thermal ellipsoids are set at a 50\% probability level.
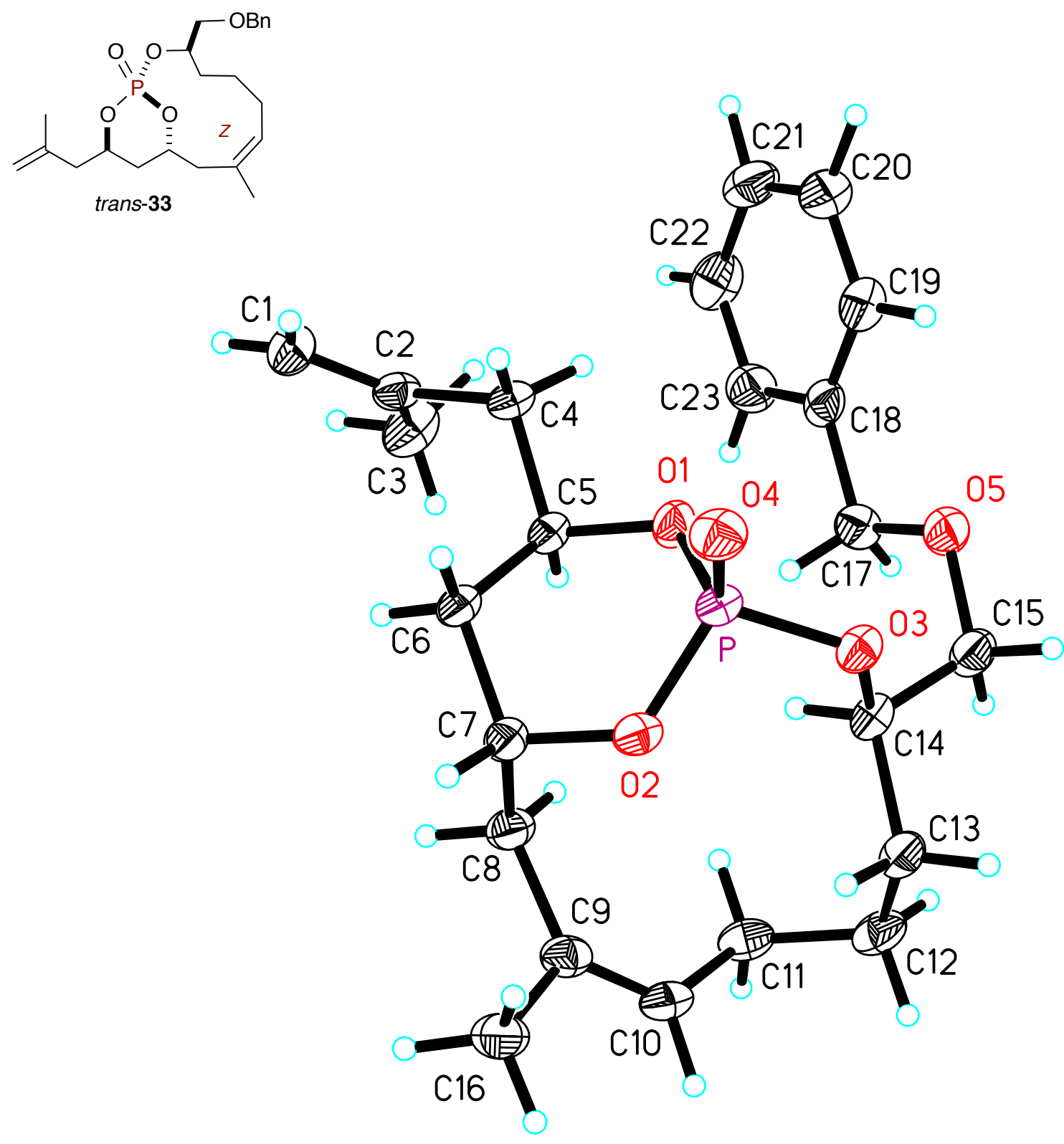
Table 3. Crystal data and structure refinement for Compound trans $-\mathbf{3 3}\left(\mathrm{C}_{23} \mathrm{H}_{33} \mathrm{O}_{5} \mathrm{P}\right)$

Identification code

Empirical formula

Formula weight

Temperature

Wavelength

Crystal system

Space group

Unit cell dimensions

Volume

$\mathrm{Z}$

Density (calculated)

Absorption coefficient

$\mathrm{F}(000)$

Crystal size

Theta range for data collection

Index ranges

Reflections collected

Independent reflections

Completeness to theta $=66.000^{\circ}$

Absorption correction

Max. and min. transmission

Refinement method

Data / restraints / parameters

Goodness-of-fit on $\mathrm{F}^{2}$

Final $\mathrm{R}$ indices [I $>2 \operatorname{sigma}(\mathrm{I})]$

$\mathrm{R}$ indices (all data)

Absolute structure parameter

Extinction coefficient

Largest diff. peak and hole
Compound trans-33

$\mathrm{C}_{23} \mathrm{H}_{33} \mathrm{O}_{5} \mathrm{P}$

420.46

100(2) K

$1.54178 \AA$

Monoclinic

P 21

$\mathrm{a}=6.0855(4) \AA \quad \alpha=90^{\circ}$

$\mathrm{b}=20.7040(13) \AA \quad \beta=107.5800(10)^{\circ}$

$\mathrm{c}=9.2928(6) \AA \quad \gamma=90^{\circ}$

1116.16(12) $\AA^{3}$

2

$1.251 \mathrm{Mg} / \mathrm{m}^{3}$

$1.342 \mathrm{~mm}^{-1}$

452

$0.270 \times 0.230 \times 0.110 \mathrm{~mm}^{3}$

5.431 to $67.909^{\circ}$

$-5<=\mathrm{h}<=7,-18<=\mathrm{k}<=24,-11<=\mathrm{l}<=10$

6459

$2986[\mathrm{R}(\mathrm{int})=0.0172]$

$98.1 \%$

Multi-scan

1.000 and 0.902

Full-matrix least-squares on $\mathrm{F}^{2}$

2986 / 1 / 394

1.069

$\mathrm{R} 1=0.0255, \mathrm{wR} 2=0.0655$

$\mathrm{R} 1=0.0255, \mathrm{wR} 2=0.0656$

0.102(7)

$\mathrm{n} / \mathrm{a}$

0.366 and -0.222 e. $\AA^{-3}$ 


\begin{tabular}{|c|c|c|c|}
\hline $\mathrm{P}-\mathrm{O}(4)$ & $1.4555(16)$ & $C(12)-C(13)$ & $1.532(4)$ \\
\hline $\mathrm{P}-\mathrm{O}(1)$ & $1.5686(17)$ & $\mathrm{C}(12)-\mathrm{H}(12 \mathrm{~A})$ & $0.96(3)$ \\
\hline $\mathrm{P}-\mathrm{O}(2)$ & $1.5686(17)$ & $\mathrm{C}(12)-\mathrm{H}(12 \mathrm{~B})$ & $1.00(3)$ \\
\hline $\mathrm{P}-\mathrm{O}(3)$ & $1.5726(17)$ & $C(13)-C(14)$ & $1.524(3)$ \\
\hline $\mathrm{O}(1)-\mathrm{C}(5)$ & $1.469(3)$ & $\mathrm{C}(13)-\mathrm{H}(13 \mathrm{~A})$ & $0.99(3)$ \\
\hline $\mathrm{O}(2)-\mathrm{C}(7)$ & $1.462(3)$ & $\mathrm{C}(13)-\mathrm{H}(13 \mathrm{~B})$ & $0.96(3)$ \\
\hline $\mathrm{O}(3)-\mathrm{C}(14)$ & $1.468(3)$ & $C(14)-C(15)$ & $1.517(3)$ \\
\hline $\mathrm{O}(5)-\mathrm{C}(17)$ & $1.411(3)$ & $\mathrm{C}(14)-\mathrm{H}(14)$ & $0.92(3)$ \\
\hline $\mathrm{O}(5)-\mathrm{C}(15)$ & $1.422(3)$ & $\mathrm{C}(15)-\mathrm{H}(15 \mathrm{~A})$ & $1.00(3)$ \\
\hline$C(1)-C(2)$ & $1.327(4)$ & $\mathrm{C}(15)-\mathrm{H}(15 \mathrm{~B})$ & $1.02(3)$ \\
\hline $\mathrm{C}(1)-\mathrm{H}(1 \mathrm{~A})$ & $0.99(4)$ & $\mathrm{C}(16)-\mathrm{H}(16 \mathrm{~A})$ & $0.95(4)$ \\
\hline $\mathrm{C}(1)-\mathrm{H}(1 \mathrm{~B})$ & $1.02(3)$ & $\mathrm{C}(16)-\mathrm{H}(16 \mathrm{~B})$ & $1.01(3)$ \\
\hline $\mathrm{C}(2)-\mathrm{C}(3)$ & $1.505(3)$ & $C(16)-H(16 C)$ & $0.99(3)$ \\
\hline$C(2)-C(4)$ & $1.507(3)$ & $C(17)-C(18)$ & $1.504(4)$ \\
\hline $\mathrm{C}(3)-\mathrm{H}(3 \mathrm{~A})$ & $0.99(4)$ & $\mathrm{C}(17)-\mathrm{H}(17 \mathrm{~A})$ & $0.95(3)$ \\
\hline $\mathrm{C}(3)-\mathrm{H}(3 \mathrm{~B})$ & $0.92(4)$ & $\mathrm{C}(17)-\mathrm{H}(17 \mathrm{~B})$ & $1.05(3)$ \\
\hline $\mathrm{C}(3)-\mathrm{H}(3 \mathrm{C})$ & $1.10(3)$ & $\mathrm{C}(18)-\mathrm{C}(19)$ & $1.380(4)$ \\
\hline$C(4)-C(5)$ & $1.523(3)$ & $C(18)-C(23)$ & $1.397(4)$ \\
\hline $\mathrm{C}(4)-\mathrm{H}(4 \mathrm{~A})$ & $0.95(3)$ & $\mathrm{C}(19)-\mathrm{C}(20)$ & $1.376(4)$ \\
\hline $\mathrm{C}(4)-\mathrm{H}(4 \mathrm{~B})$ & $1.01(3)$ & $\mathrm{C}(19)-\mathrm{H}(19)$ & $1.00(4)$ \\
\hline$C(5)-C(6)$ & $1.517(3)$ & $\mathrm{C}(20)-\mathrm{C}(21)$ & $1.385(4)$ \\
\hline $\mathrm{C}(5)-\mathrm{H}(5 \mathrm{~A})$ & $0.96(3)$ & $\mathrm{C}(20)-\mathrm{H}(20)$ & $1.05(4)$ \\
\hline$C(6)-C(7)$ & $1.530(4)$ & $\mathrm{C}(21)-\mathrm{C}(22)$ & $1.380(5)$ \\
\hline $\mathrm{C}(6)-\mathrm{H}(6 \mathrm{~A})$ & $0.98(3)$ & $\mathrm{C}(21)-\mathrm{H}(21)$ & $1.00(4)$ \\
\hline $\mathrm{C}(6)-\mathrm{H}(6 \mathrm{~B})$ & $0.98(3)$ & $\mathrm{C}(22)-\mathrm{C}(23)$ & $1.392(4)$ \\
\hline$C(7)-C(8)$ & $1.524(3)$ & $\mathrm{C}(22)-\mathrm{H}(22)$ & $0.97(5)$ \\
\hline $\mathrm{C}(7)-\mathrm{H}(7 \mathrm{~A})$ & $1.00(3)$ & $\mathrm{C}(23)-\mathrm{H}(23)$ & $0.96(4)$ \\
\hline$C(8)-C(9)$ & $1.513(3)$ & $\mathrm{O}(4)-\mathrm{P}-\mathrm{O}(1)$ & $114.73(11)$ \\
\hline $\mathrm{C}(8)-\mathrm{H}(8 \mathrm{~A})$ & $0.95(3)$ & $\mathrm{O}(4)-\mathrm{P}-\mathrm{O}(2)$ & 114.98(9) \\
\hline $\mathrm{C}(8)-\mathrm{H}(8 \mathrm{~B})$ & $0.98(3)$ & $\mathrm{O}(1)-\mathrm{P}-\mathrm{O}(2)$ & $106.23(9)$ \\
\hline $\mathrm{C}(9)-\mathrm{C}(10)$ & $1.334(4)$ & $\mathrm{O}(4)-\mathrm{P}-\mathrm{O}(3)$ & $111.06(9)$ \\
\hline$C(9)-C(16)$ & $1.514(4)$ & $\mathrm{O}(1)-\mathrm{P}-\mathrm{O}(3)$ & 104.66(9) \\
\hline $\mathrm{C}(10)-\mathrm{C}(11)$ & $1.498(4)$ & $\mathrm{O}(2)-\mathrm{P}-\mathrm{O}(3)$ & $104.15(9)$ \\
\hline $\mathrm{C}(10)-\mathrm{H}(10)$ & $0.97(3)$ & $\mathrm{C}(5)-\mathrm{O}(1)-\mathrm{P}$ & $123.52(14)$ \\
\hline $\mathrm{C}(11)-\mathrm{C}(12)$ & $1.537(4)$ & $\mathrm{C}(7)-\mathrm{O}(2)-\mathrm{P}$ & $123.91(15)$ \\
\hline $\mathrm{C}(11)-\mathrm{H}(11 \mathrm{~A})$ & $0.96(3)$ & $\mathrm{C}(14)-\mathrm{O}(3)-\mathrm{P}$ & $118.36(14)$ \\
\hline $\mathrm{C}(11)-\mathrm{H}(11 \mathrm{~B})$ & $1.00(3)$ & $\mathrm{C}(17)-\mathrm{O}(5)-\mathrm{C}(15)$ & $112.4(2)$ \\
\hline
\end{tabular}




\begin{tabular}{|c|c|c|c|}
\hline $\mathrm{C}(2)-\mathrm{C}(1)-\mathrm{H}(1 \mathrm{~A})$ & $118.9(19)$ & $\mathrm{C}(9)-\mathrm{C}(8)-\mathrm{H}(8 \mathrm{~B})$ & $109.5(17)$ \\
\hline $\mathrm{C}(2)-\mathrm{C}(1)-\mathrm{H}(1 \mathrm{~B})$ & $116(2)$ & $\mathrm{C}(7)-\mathrm{C}(8)-\mathrm{H}(8 \mathrm{~B})$ & $111.7(16)$ \\
\hline $\mathrm{H}(1 \mathrm{~A})-\mathrm{C}(1)-\mathrm{H}(1 \mathrm{~B})$ & $125(3)$ & $\mathrm{H}(8 \mathrm{~A})-\mathrm{C}(8)-\mathrm{H}(8 \mathrm{~B})$ & $107(2)$ \\
\hline $\mathrm{C}(1)-\mathrm{C}(2)-\mathrm{C}(3)$ & $122.7(2)$ & $\mathrm{C}(10)-\mathrm{C}(9)-\mathrm{C}(8)$ & $124.6(2)$ \\
\hline$C(1)-C(2)-C(4)$ & $121.5(2)$ & $C(10)-C(9)-C(16)$ & $120.0(2)$ \\
\hline $\mathrm{C}(3)-\mathrm{C}(2)-\mathrm{C}(4)$ & $115.8(2)$ & $C(8)-C(9)-C(16)$ & $115.4(2)$ \\
\hline $\mathrm{C}(2)-\mathrm{C}(3)-\mathrm{H}(3 \mathrm{~A})$ & $111(2)$ & $\mathrm{C}(9)-\mathrm{C}(10)-\mathrm{C}(11)$ & $129.8(2)$ \\
\hline $\mathrm{C}(2)-\mathrm{C}(3)-\mathrm{H}(3 \mathrm{~B})$ & $112(2)$ & $\mathrm{C}(9)-\mathrm{C}(10)-\mathrm{H}(10)$ & $117.8(17)$ \\
\hline $\mathrm{H}(3 \mathrm{~A})-\mathrm{C}(3)-\mathrm{H}(3 \mathrm{~B})$ & $108(3)$ & $\mathrm{C}(11)-\mathrm{C}(10)-\mathrm{H}(10)$ & $112.3(16)$ \\
\hline $\mathrm{C}(2)-\mathrm{C}(3)-\mathrm{H}(3 \mathrm{C})$ & $106.9(15)$ & $C(10)-C(11)-C(12)$ & $111.3(2)$ \\
\hline $\mathrm{H}(3 \mathrm{~A})-\mathrm{C}(3)-\mathrm{H}(3 \mathrm{C})$ & 109(3) & $\mathrm{C}(10)-\mathrm{C}(11)-\mathrm{H}(11 \mathrm{~A})$ & $112.6(14)$ \\
\hline $\mathrm{H}(3 \mathrm{~B})-\mathrm{C}(3)-\mathrm{H}(3 \mathrm{C})$ & $110(3)$ & $\mathrm{C}(12)-\mathrm{C}(11)-\mathrm{H}(11 \mathrm{~A})$ & $108.4(14)$ \\
\hline$C(2)-C(4)-C(5)$ & $113.41(19)$ & $\mathrm{C}(10)-\mathrm{C}(11)-\mathrm{H}(11 \mathrm{~B})$ & $107.9(17)$ \\
\hline $\mathrm{C}(2)-\mathrm{C}(4)-\mathrm{H}(4 \mathrm{~A})$ & $109.0(18)$ & $\mathrm{C}(12)-\mathrm{C}(11)-\mathrm{H}(11 \mathrm{~B})$ & 109.7(17) \\
\hline $\mathrm{C}(5)-\mathrm{C}(4)-\mathrm{H}(4 \mathrm{~A})$ & $106.7(18)$ & $\mathrm{H}(11 \mathrm{~A})-\mathrm{C}(11)-\mathrm{H}(11 \mathrm{~B})$ & $107(2)$ \\
\hline $\mathrm{C}(2)-\mathrm{C}(4)-\mathrm{H}(4 \mathrm{~B})$ & $110.2(16)$ & $C(13)-C(12)-C(11)$ & $113.9(2)$ \\
\hline $\mathrm{C}(5)-\mathrm{C}(4)-\mathrm{H}(4 \mathrm{~B})$ & $104.5(17)$ & $\mathrm{C}(13)-\mathrm{C}(12)-\mathrm{H}(12 \mathrm{~A})$ & $106.9(17)$ \\
\hline $\mathrm{H}(4 \mathrm{~A})-\mathrm{C}(4)-\mathrm{H}(4 \mathrm{~B})$ & $113(2)$ & $\mathrm{C}(11)-\mathrm{C}(12)-\mathrm{H}(12 \mathrm{~A})$ & $107.8(17)$ \\
\hline $\mathrm{O}(1)-\mathrm{C}(5)-\mathrm{C}(6)$ & $110.14(18)$ & $\mathrm{C}(13)-\mathrm{C}(12)-\mathrm{H}(12 \mathrm{~B})$ & $109.8(17)$ \\
\hline $\mathrm{O}(1)-\mathrm{C}(5)-\mathrm{C}(4)$ & $104.82(18)$ & $\mathrm{C}(11)-\mathrm{C}(12)-\mathrm{H}(12 \mathrm{~B})$ & $110.5(17)$ \\
\hline$C(6)-C(5)-C(4)$ & $114.04(19)$ & $\mathrm{H}(12 \mathrm{~A})-\mathrm{C}(12)-\mathrm{H}(12 \mathrm{~B})$ & $108(2)$ \\
\hline $\mathrm{O}(1)-\mathrm{C}(5)-\mathrm{H}(5 \mathrm{~A})$ & $108.3(15)$ & $C(14)-C(13)-C(12)$ & $113.2(2)$ \\
\hline $\mathrm{C}(6)-\mathrm{C}(5)-\mathrm{H}(5 \mathrm{~A})$ & $108.6(14)$ & $\mathrm{C}(14)-\mathrm{C}(13)-\mathrm{H}(13 \mathrm{~A})$ & $106(2)$ \\
\hline $\mathrm{C}(4)-\mathrm{C}(5)-\mathrm{H}(5 \mathrm{~A})$ & $110.8(16)$ & $\mathrm{C}(12)-\mathrm{C}(13)-\mathrm{H}(13 \mathrm{~A})$ & 109.3(19) \\
\hline$C(5)-C(6)-C(7)$ & $114.07(19)$ & $\mathrm{C}(14)-\mathrm{C}(13)-\mathrm{H}(13 \mathrm{~B})$ & $109.9(16)$ \\
\hline $\mathrm{C}(5)-\mathrm{C}(6)-\mathrm{H}(6 \mathrm{~A})$ & $106.6(16)$ & $\mathrm{C}(12)-\mathrm{C}(13)-\mathrm{H}(13 \mathrm{~B})$ & $110.6(16)$ \\
\hline$C(7)-C(6)-H(6 A)$ & $108.5(18)$ & $\mathrm{H}(13 \mathrm{~A})-\mathrm{C}(13)-\mathrm{H}(13 \mathrm{~B})$ & $107(2)$ \\
\hline $\mathrm{C}(5)-\mathrm{C}(6)-\mathrm{H}(6 \mathrm{~B})$ & $109.5(17)$ & $\mathrm{O}(3)-\mathrm{C}(14)-\mathrm{C}(15)$ & $107.04(19)$ \\
\hline $\mathrm{C}(7)-\mathrm{C}(6)-\mathrm{H}(6 \mathrm{~B})$ & 107.7(19) & $\mathrm{O}(3)-\mathrm{C}(14)-\mathrm{C}(13)$ & $108.98(18)$ \\
\hline $\mathrm{H}(6 \mathrm{~A})-\mathrm{C}(6)-\mathrm{H}(6 \mathrm{~B})$ & $110(2)$ & $C(15)-C(14)-C(13)$ & $112.5(2)$ \\
\hline $\mathrm{O}(2)-\mathrm{C}(7)-\mathrm{C}(8)$ & $106.8(2)$ & $\mathrm{O}(3)-\mathrm{C}(14)-\mathrm{H}(14)$ & $104.7(17)$ \\
\hline $\mathrm{O}(2)-\mathrm{C}(7)-\mathrm{C}(6)$ & $110.37(18)$ & $\mathrm{C}(15)-\mathrm{C}(14)-\mathrm{H}(14)$ & $112.3(17)$ \\
\hline $\mathrm{C}(8)-\mathrm{C}(7)-\mathrm{C}(6)$ & $115.2(2)$ & $\mathrm{C}(13)-\mathrm{C}(14)-\mathrm{H}(14)$ & $110.9(18)$ \\
\hline $\mathrm{O}(2)-\mathrm{C}(7)-\mathrm{H}(7 \mathrm{~A})$ & $107.2(18)$ & $\mathrm{O}(5)-\mathrm{C}(15)-\mathrm{C}(14)$ & $114.8(2)$ \\
\hline $\mathrm{C}(8)-\mathrm{C}(7)-\mathrm{H}(7 \mathrm{~A})$ & 109.7(17) & $\mathrm{O}(5)-\mathrm{C}(15)-\mathrm{H}(15 \mathrm{~A})$ & 105.1(19) \\
\hline $\mathrm{C}(6)-\mathrm{C}(7)-\mathrm{H}(7 \mathrm{~A})$ & $107(2)$ & $\mathrm{C}(14)-\mathrm{C}(15)-\mathrm{H}(15 \mathrm{~A})$ & $110.2(19)$ \\
\hline $\mathrm{C}(9)-\mathrm{C}(8)-\mathrm{C}(7)$ & $112.9(2)$ & $\mathrm{O}(5)-\mathrm{C}(15)-\mathrm{H}(15 \mathrm{~B})$ & $110.7(18)$ \\
\hline $\mathrm{C}(9)-\mathrm{C}(8)-\mathrm{H}(8 \mathrm{~A})$ & 111.6(19) & $\mathrm{C}(14)-\mathrm{C}(15)-\mathrm{H}(15 \mathrm{~B})$ & $105.6(16)$ \\
\hline $\mathrm{C}(7)-\mathrm{C}(8)-\mathrm{H}(8 \mathrm{~A})$ & $103.9(17)$ & $\mathrm{H}(15 \mathrm{~A})-\mathrm{C}(15)-\mathrm{H}(15 \mathrm{~B})$ & $110(3)$ \\
\hline
\end{tabular}




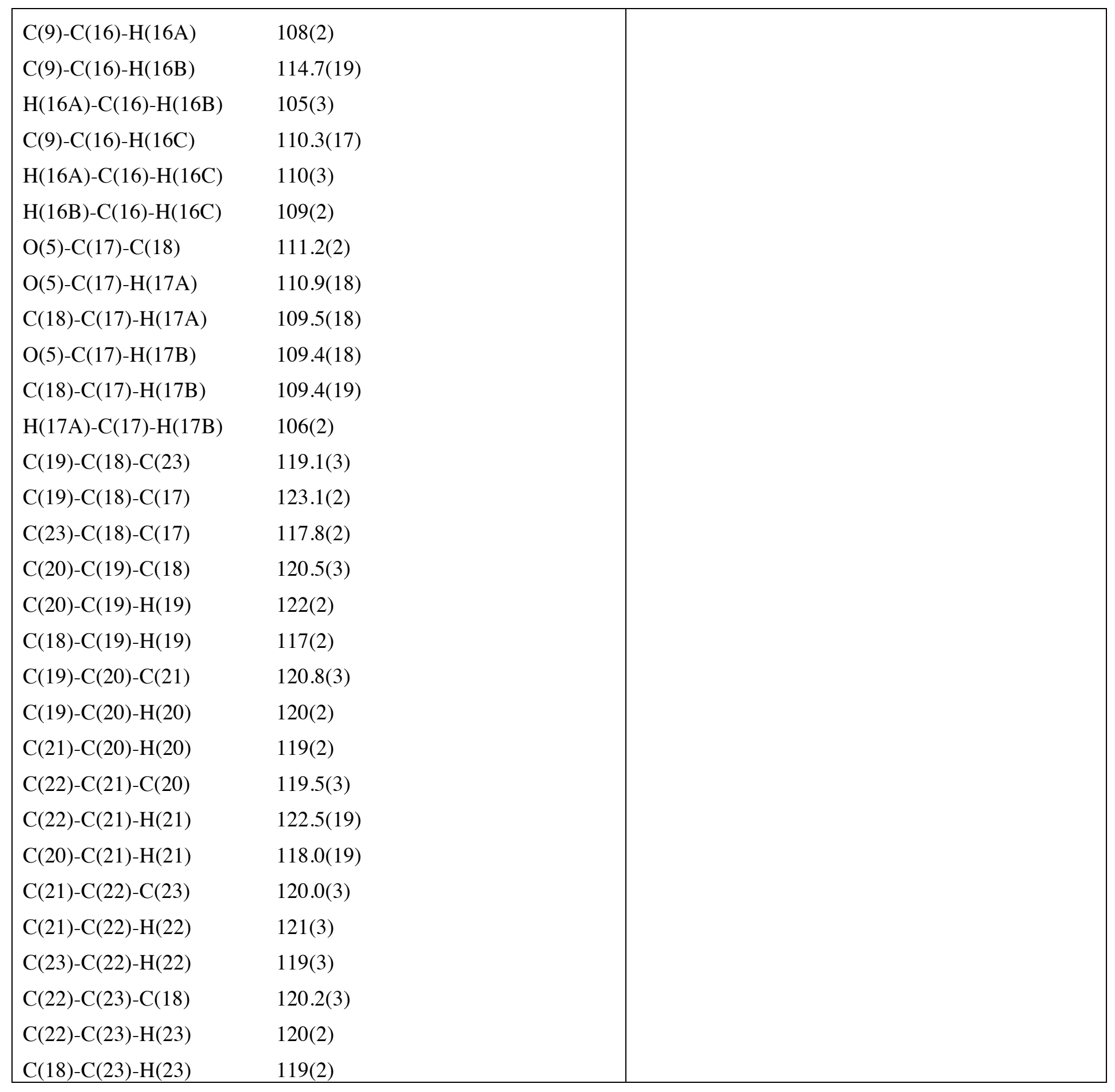

